\title{
Selection of Two Identical Pictures from a Group of Similar Ones II: Changes in Ongoing EEG Activity
}

J. Petrek

Institute of Physiology, Faculty of Medicine, Palacky University

Correspondence to: Josef Petrek, MD

Institute of Physiology

Palacky University

Hnevotinska 3,

77515 Olomouc

Czech Republic

Key words:

cognitive task, searching for identical pictures, power EEG spectrum, site of recording electrode, personality traits

Activitas Nervosa Superior 2008;50:1-2,3-8

\section{Abstract}

The paper presents finding of an experiment the aim of which was to judge the impact of an instant cognitive activity (identical pictures searching) on behavior of ongoing EEG activity. Two types of mental task were used: The first - passive watching of a blank white oval and the second - active searching for identical pictures in a group of similar nine line-drawings of living individuals or inanimate objects filling out the white oval.

Presented results showed that higher mental load pertinent to active searching for identical pictures in a group of similar pictures results in the prominent event-related desynchronization (ERD) - the mean Total Power value, a quantitative measure of ERD extent, in comparison with reference level (passive watching) was lower while solving the mental task.

The results also showed that the actual mental task performance affects the ERD only at some scalp-recording sites. The mean EEG Total Power significantly decreases at parietal and frontal scalp-recording sites whereas the significant decrease of the Frequency at Maximum Power involves occipital scalp electrodes, too.

Our results also demonstrated that some subject's personality traits (moderation, openness and extraversion) affect the actual decrease/increase in size of Frequency at Maximum Power during active mental task solving.

Presented findings point at the high suitability of the ERD method to uncover differences in people's brain activation patterns when engaged in performing cognitively demanding tasks. 
A large number of papers published in the last three decades show that research of brain oscillations during cognitive and motor brain functions brings valuable information allowing us to better understand the functional organization of the brain and its role in organizing an optimal behavior of an organism.

Sensory and cognitive processing results not only in an event-related potential (ERP), but also in a change in the ongoing EEG. The former potentials are of short duration $(-200-300 \mathrm{~ms})$ and reflect direct neuronal activation; the latter represents a short-lasting decrease/increase in rhythmic activity (event - related desynchronization /synchronization or ERD/ERS) that occurs in relation to an event (Pfurtscheller and Aranibar 1977). The ERD and ERS reflect the dynamics of neuronal networks and that is why they are recognized, together with ERP, as a valuable tool in neurocognitive reseach. For further details see for example Pfurtscheller (1977), Lopes da Silva (1991), Morrell (1996), Klimesch (1999), Pfurtscheller and Lopes da Silva (1999), Pfurtscheller (1992, 2001, 2003), Mazaheri (2005).

The ERD is not only an electrophysiological correlate of cortical activation related to stimulus processing but also characteristic of cortical areas preparing to process sensory information or ready to execute a motor command. It is therefore not surprising that many cognitive psychologists and neuroscientists combine psychological testing or solving special cognitive tasks with simultaneous registration of brain electrical activity. The review of Klimesch (1999) and monographs of Faber (2001, 2005) and especially papers presented in the book "EventRelated Dynamics of Brain Oscillations", edited by Neuper C. and Klimesch W. in 2006 confirm it.

The aim of our experiment was to judge the impact of an instant cognitive activity (selection of two identical pictures in a group of similar pictures) on brain potentials - ERP and an ongoing EEG. All visual stimuli (drawing of living creatures or common objects) were taken from Matching Familiar Test TE-NA-ZO (Müllner et al. 1986). Our earlier paper (Petrek 2007) was addressed to an analysis of ERPs dynamics during selection of two identical pictures. The present paper deals with the EEG changes in the course of a cognitive problems solving. Attention is paid especially to the dynamics of ERD and its relation to the site of recording electrode and to the participants' personality traits.

\section{METHODS}

The experimental paradigm, procedures, features of visual stimuli and method of data acquisition were described in detail in our earlier paper (Petrek 2007). Here only the principles of the data analysis are described.

SciWorks version 5 with DataWave CP Analysis Modules and Data Editing Software were used for an off-line analysis of experimental data. The analysis ran as follows: A visual elimination of distorted records, sorting records into groups according the type of mental task, digital filtering of records (bandpass 1-30 Hz), and cutting digitally filtered records for the Fast Fourier Transform analysis (FFT) - the starting points of the cut segments of EEG record were time-locked to the photodiode signal.

For each experimental task and everybody's subject the SciWork FFT Analysis module calculated the total power histograms from a fixed frequency band $(3-20 \mathrm{~Hz}$ or $8-13$ $\mathrm{Hz}$ ) for each 3-second EEG sample with $50 \%$ overlay and all electrodes. From the histograms the software derived two FFT single values - Total Power and Frequency at Maximum Power, averaged either of them separately and actual average numerical values visualized in a spreadsheet format on the screen DataPad Plugin module. Subsequent statistical data analyses were accomplished with the StatSoft software package (StatSoft, Tulsa, OK).

The average decrease in Total Power and Frequency at Maximum Power expressed as the percentage of decrease in TP/FMP within the frequency band of interest in the period of the mental task as compared to the reference interval (passive watching of a blank white oval) was used to quantify ERD/ERS and the EEG frequency change within the frequency band of interest.

\section{RESULTS}

Table 1 summarizes the basic statistical characteristics of tested parameters in our experiment. From it follows that the type of the experimental task (passive watching of a blank white oval or active searching for identical pictures in a group of similar ones) determines the mean values of Total Power calculated for the 3 to $20 \mathrm{~Hz}$ EEG frequency bands (TP) or for the 8 to $13 \mathrm{~Hz}$ EEG frequency bands (TPA). The experimental task affects also the mean Frequency at Maximum Power computed for the former frequency band (FM), but the Frequency at Maximum Power of the latter band (FMA), that is the alpha band, shows no such dependence.

The mean TP and TPA values as well as FM values are lower during mental task solving (active searching for identical pictures in a group of similar ones) and higher during passive watching of a blank white oval (reference level). However, this is not valid for FMA (the average Frequency at Maximum Power of the 8 to $13 \mathrm{~Hz}$ EEG 
frequency bands) - the FMA means do not differ from each other. The results of the Simple Factorial ANOVA with Repeated Measures confirm these conclusions (for details see Figure 1).
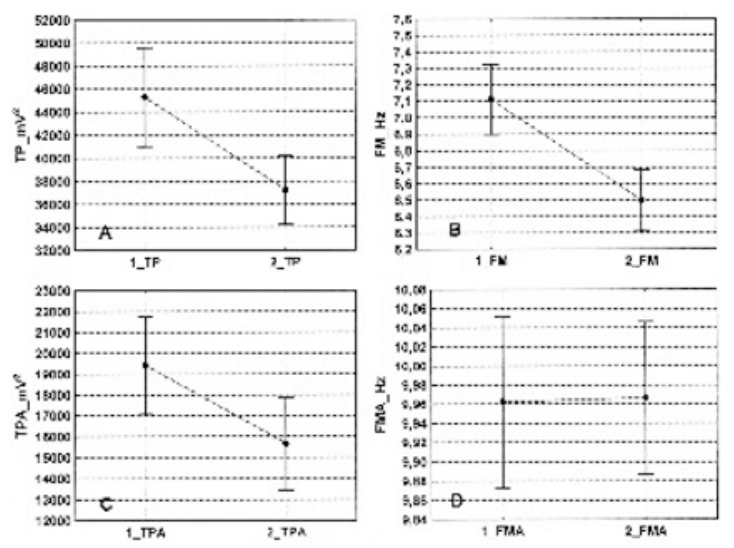

Fig. 1 Comparison of FFT parameters in two experimental situations.

A - TP; Current effect: $F(1,204)=65.435, p=.0000$.

$B$ - TPA; Current effect: $F(1,204)=17.229, p=.0000$.

$C$ - FM; Current effect: $F(1,204)=108.92, p=.0000$.

$D$ - FMA; Current effect: $F(1,204)=.02271, p=.8803$.

Black circles - mean, vertical bars denote 0.95 confidence intervals.

For further details see the text to Table 1.

ANOVA results also showed that besides the experimental situation the site of the scalp-recording electrode affects the mean TP, TPA and FM values too $[\operatorname{FTP}(11$, $204)=6.4028, \quad \mathrm{p}=.00000], \quad[\operatorname{FTPA}(11,204)=4.0413$, $\mathrm{p}=.00002],[\operatorname{FFM}(11,204)=3.6749, \mathrm{p}=.00009]$. The post hoc comparison of means (Duncan's test) showed that the mean TP values are significantly higher at F3, F4, P3 and $\mathrm{P} 4$ while the TPA values only at P3 and P4. Contrary to $\mathrm{TP}$, the mean MF values reach maximum not only at all parietal but also at occipital scalp-recording electrodes in both hemispheres. The mean FMA values did not show any dependence on the site of recording electrode $[$ FFMA $(11,204)=0.85800, \mathrm{p}=.58246]$.

The statistically significant interactions between tested variables (TP, TPA, MF and FMA) and the independent variables (the type of mental task and the site of scalprecording electrode) were also established (see Figure 2).

From Figure $2 \mathrm{~A}$ follows that increasing mental load significantly decreases TP at P3, P4, F3 and F4 scalprecording sites. The TP in other electrodes did not show such dependence on the task demands - there are no significant differences between the compared means (i. e., between mean TP values during passive watching of a blank white oval and mean TP values during active searching for identical pictures in a group of similar ones).
A higher mental load significantly decreases also the mean MF values at all parietal and occipital electrodes in both hemispheres with the exception of CP5 electrode. It also decreases mean FMA value at $\mathrm{O} 2$ and increases it at F4 electrodes (Figure 2C and D). The decrease in TPA is restricted only to the parietal scalp-recording electrodes (Figure 2B).

The average decrease in TP and MF expressed as percentage of decrease in band power/frequency during a mental task as compared to the reference interval (passive watching of a blank white oval) ranged from 7 to 10 per cent, with the exception of FMA - the latter value ranged around zero (see Table 1). The average percentage of decrease in TP and FM reached statistical significance in both cases ( $\mathrm{t}$-test for a single mean).

The results of analysis of variance (Repeated Measures ANOVA) and standard regression analysis confirmed our assumption that some subjects' personality traits could affect the actual decrease/increase size of the tested parameters during mental task solving. It has been shown that the five FPI subject's personality features from the thirteen established affect the extent in decrease/increase of FM and FMA. They are especially moderation (FPI 8), openness (FPI 9) and extraversion (E). The first of them was related to FM, the other two to FMA. The TP and TPA variables did not show any relation to FPI subject's personality traits.

\begin{tabular}{|c|c|c|c|c|c|}
\hline Parameter & $\mathrm{N}$ & Mean & $\begin{array}{l}\text { Confidence } \\
(+95.00 \\
\%)\end{array}$ & $\begin{array}{c}\text { Interval } \\
(-95.00 \\
\%)\end{array}$ & St.Deviation \\
\hline 1_TP & 216 & $\begin{array}{r}45 \\
313,05\end{array}$ & 40512,51 & $\begin{array}{r}50 \\
113,58 \\
\end{array}$ & 35794,53 \\
\hline 2_TP & 216 & $\begin{array}{r}37 \\
247,43 \\
\end{array}$ & 33877,78 & $\begin{array}{r}40 \\
617,07 \\
\end{array}$ & 25125,33 \\
\hline$\%$ _TP & 216 & $-10,47$ & $-13,04$ & $-7,89$ & 19,20 \\
\hline 1.FM & 216 & 7,11 & 6,88 & 7,34 & 1,72 \\
\hline $2 \mathrm{FM}$ & 216 & 6,50 & 6,30 & 6,69 & 1,45 \\
\hline$\%$ FM & 216 & $-7,36$ & $-8,87$ & $-5,85$ & 11,25 \\
\hline 1_TPA & 216 & $\begin{array}{r}19 \\
436,13 \\
\end{array}$ & 16853,03 & $\begin{array}{r}22 \\
019,23 \\
\end{array}$ & 19260,56 \\
\hline 2_TPA & 216 & $\begin{array}{r}15 \\
669,62 \\
\end{array}$ & 13387,97 & $\begin{array}{r}17 \\
951,27 \\
\end{array}$ & 17012,79 \\
\hline$\%$ TPA & 216 & $-17,23$ & $-19,58$ & $-14,88$ & 17,51 \\
\hline 1.FMA & 216 & 9,96 & 9,87 & 10,05 & 0,68 \\
\hline 2. FMA & 216 & 9,97 & 9,89 & 10,05 & 0,59 \\
\hline$\%$ FMA & 216 & $-0,21$ & $-0,76$ & 0,35 & 4,13 \\
\hline
\end{tabular}

Table 1 Basic statistical characteristics of tested parameters

1_TP (1_FM) - Total Power - in $m V^{2}$ (Frequency at Maximum Powerin $\mathrm{Hz}$ ) of the 3 to $20 \mathrm{~Hz} E E G$ frequency bands during a reference task.

2_TP (2_FM) - Total Power (Frequency at Maximum Power) of the 3 to $20 \mathrm{~Hz} E E \mathrm{G}$ frequency bands during active mental task solving. 
1_TPA (1_FMA) - Total Power (Frequency at Maximum Power) of the 8 to $13 \mathrm{~Hz} E E G$ frequency bands during a reference task. 2_TPA (2_FMA) - Total Power (Frequency at Maximum Power) of the 8 to $13 \mathrm{~Hz} E E G$ frequency bands during active mental task solving.

The scatterplots in Figure 3 graphically demonstrate the mutual relationship between FPI subjects' score and the mean decrease/increase in size of FM/FMA (in percentage) during its intensive cognitive activity.

\section{DISCUSSION}

In our paper (Petrek 2007) it was shown that cognitive processes underlying successful selection of two identical pictures from a group of similar pictures affect the activity of systems giving rise to ERPs. The amplitude changes of individual ERP components revealed it. The present paper extends this conclusion showing that cognitive processes also result in a change in the ongoing EEG activity. The short-lasting and localized amplitude decrease in rhythmic activity (ERD) and the change of EEG frequency are among the most frequent changes.
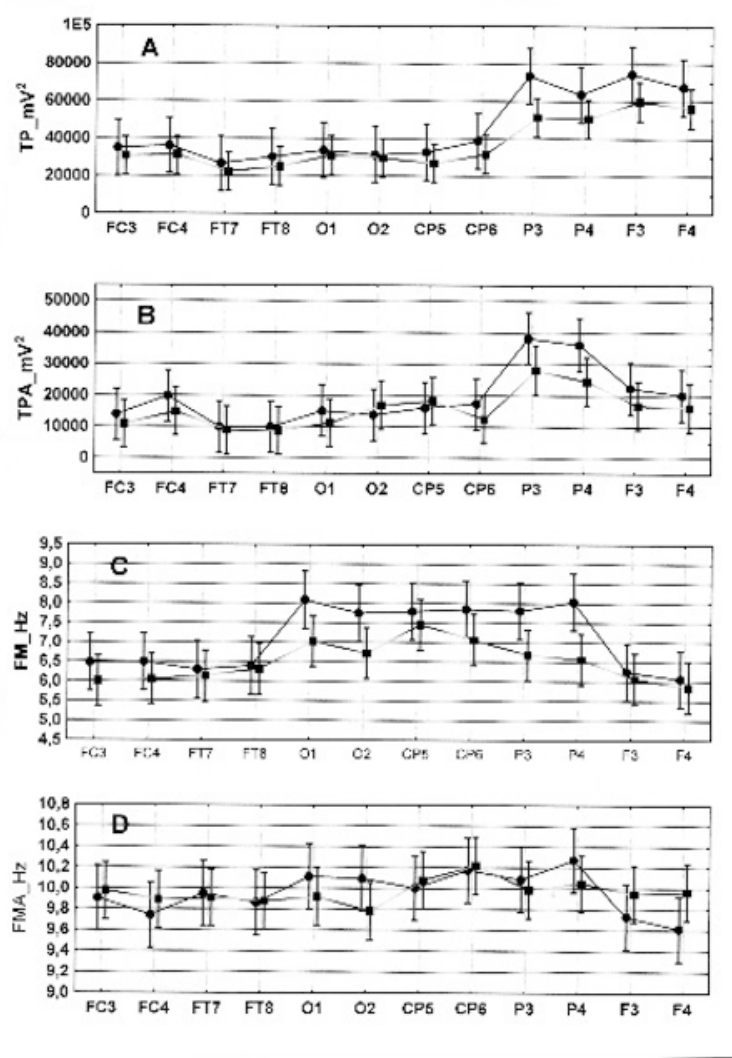

Fig. 2 Interaction between FFT parameters and the site of scalprecording electrode.

$A-T P$ vs. electrode location; Current effect: $F(11,204)=2.9744, p=$ .0011.

$B-T P A$ vs. electrode location, Current effect $F(11,204)=$
$B-F M$ vs. electrode location; Current effect: $F(11,2004)=5.1749, p=$ .0000 .

$C-F M A$ vs. electrode location; Current effect: $F(11,204)=3.6676, p=$ .0001 .

Black circles and full lines - reference task, black squares and dotted lines - active mental task solving. For the other one see the text to Table 1 and Figure 1.

The presented results show that a higher mental load pertinent to active searching for identical pictures in a group of similar pictures results in a prominent ERD -the mean TP value, a quantitative measure of ERD extent, in comparison with reference level was lower during the mental task, both for the wide $(3-20 \mathrm{~Hz})$ and the narrow $(8-13 \mathrm{~Hz})$ EEG frequency bands. These findings correspond with those of many other authors (for details see for example Klimesch - 1996, Pfurtscheller - 2001, Faber - 2005). It has to be so because maximal readiness and optimal excitability of neural structures (the extent of ERD represents their objective measure) is a prerequisite of successful processing of information in specific brain systems during the solving of a cognitive task.

It is accepted that ERD is not only an electrophysiological correlate of cortical activation to stimulus processing but also a characteristic of cortical areas preparing to process sensory information or ready to execute a motor command (Pfurtscheller 1992, Pfurtscheller et al. 2000). From this follows that during a cognitive task alpha ERD should be topographically localized over the corresponding brain areas involved with a specific task.

The results of our experiment are not in a fundamental conflict with this assumption. It follows from them that the actual mental task performance (selection of two identical pictures from a group of similar pictures) affects differently the mean EEG Total Power or the extent of ERD only in some scalp-recording sites. The mean TP within 3 to $20 \mathrm{~Hz}$ EEG frequency bands significantly decreases in parietal (P3, P4) and frontal (F3, F4) scalp recording sites whereas the Total Power of the alpha band (TPA) decreases in parietal recording sites only.. The mean TPA values of remaining electrodes, including occipital electrodes, do not show any dependence on the mental task performance.

The latter statement is somewhat surprising because occipital brain areas are certainly engaged in the processing of visual information pertinent to mental task solving. In this connection, however, it should be emphasized that evaluation of another dependent variable - the Frequency at Maximum Power of the 3 to $20 \mathrm{~Hz}$ EEG frequency bands, unambiguously proved the role of the occipital cortex in processing of visual information 
during mental task solving. The MFA was significantly decreased in occipital scalp electrodes.
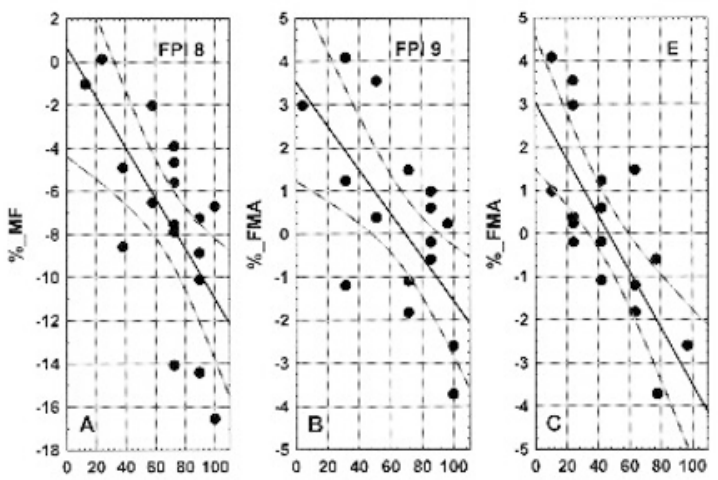

Fig. 3 Two-dimensional scatter plots: FPI score (abscissa) vs. FFT parameters

$A-F P I 8$ (moderation) vs. FM; $r=-.6023, p=.0082$.

$B-F P I 9$ (openness) vs. FMA, $r=-.6569, p=.0031$.

$C-E$ (extroversion) vs. FMA, $r=.7616, p=.0002$.

Full lines - regression lines, dotted lines - $95 \%$ confidence interval.

It appears that both FFT dependent variables - Total Power and Frequency at Maximum Power - used in our experiment as indicators of momentary excitability of neural structures have different predictive values. The FM was more sensitive and it told more accurately about engagement of different cortical areas in the processing of visual information during active mental task solving. The lower predicative value of the TP in our experiment is probably related to the method used to measure this parameter - averaging of the EEG signal, which we used, might mask the dynamics of the tested parameter and erase possible differences.

Last but not least, our results also show that some subjects' personality traits (moderation, openness and extroversion) affect the actual decrease/increase in size of Frequency at Maximum Power during active mental task solving. The influence of the personality dimension, extraversion/introversion, on the extent an topographical distribution of ERD in subjects engaged in cognitive information processing was also described by Fink (2005) and Fink et al. $(2005,2008)$. The paper by Jausovec (2000) studying the differences in cognitive processes related to creativity and intelligence by using EEG coherence and power measures should be mentioned here too. All this indicates that subjects' personality traits ought to be taken into account in the evaluation of cognitive information processing in the brain.

In conclusion we can say that our findings point at the high suitability of the ERD method for uncovering differences in brain activation patterns when people are engaged in performing cognitively demanding tasks.

\section{REFERENCES}

Faber J (2000). Electroencephalography and psychophysiology (in Czech). Praha: ISV, $170 \mathrm{p}$.

Faber J (2005). QEEG: Correlation of EEG analysis with psychological tests. Praha: Galen, $191 \mathrm{p}$.

Fink A (2005). Event-related desynchronization in the EEG during emotional and cognitive processing: Differential effects of extraversion. Biol. Psychol. 70 : 152-160.

Fink A, Grabner RH, Neuper C, Neubauer AC(2005). Extraversion and cortical activation during memory performance. Int. J. Psychophysiol. 56 : 129-141.

Fink A, Neubauer AC (2008) Eysenck meets Martindale The relationship between extraversion and originality from the neuroscientific perspective. Pers. Indiv. Dif. 44 : 299-310

Jausovec $\mathbf{N}$ (2000). Differences in cognitive processes between gifted, intelligent, creative, and average individuals while solving complex problems: An EEG study. Intelligence 28 : 213-237.

Klimesch W (1999). EEG alpha and theta oscillation reflect cognitive and memory performance: a review and analysis. Brain Res. Rev. 29 : 169-195.

Lopes da Silva FH (1991). Neural mechanisms underlying brain waves: from neural membranes to networks. Electroenceph. clin Neurophysiol. $79: 81-93$.

Mazaher A, Picton TW (2005). EEG spectral dynamics during discrimination of auditory and visual targets. Cogn. Brain Res. 24 : 8196.

Morrel LK (1996). Some characteristics of stimulus-provoked alpha activity. Electroenceph. clin Neurophysiol. 21 : 552-561.

Müllner J, Pufflerova S, Czurma L (1986). Matching Familiar Figures Test TE-NA-ZO for Adolescents and Adults (in Slovak). Bratislava: Psychodiagnosticke a didakticke testy.

Neuper C, Klimesch W (Eds) (2006). Event-related dynamics of brain oscillations. Amsterdam: Elsevier, $464 \mathrm{p}$.

Petrek J (2007). A selection of two identical pictures from a group of similar ones: I. Event-related potentials dynamics. Act Nerv Super 49 :1-2, 93-100.

Pfurtscheller G (1977). Graphical display and statistical evaluation of event-related desynchronization (ERD). Electroenceph. clin Neurophysiol. 43 : 757-760.

Pfurtscheller G (1992). Event-related synchronization (ERS): an electrophysiological correlate of cortical areas at rest. Electroenceph. clin. Neurophysiol. 83 : 62-69.

Pfurtscheller G (2001): Functional brain imaging based on ERD/ERS. Vision Res. 41 : 1257-1260. 
Pfurtscheller G (2001): Induced oscillations in the alpha band: Functional meaning. Epilepsia 44 (Suppl. 12) : 2-8.

Pfurtscheller G, Aranibar A (1977). Event-related cortical desynchronization detected by power measurements of scalp EEG. Electroenceph. clin Neurophysiol. 42 : 817-826, 1977.

Pfurtscheller G, Neuper C, Mohl W (1994). Event-related desynchronization (ERD) during visual processing. Int. J. Psychophysiol. 16 : 147-153.

Pfurtscheller G, Lopes da Silva FH (1999). Event related EEG/MEG synchronization and desynchronization : basic principles. Clin. Neurophysiol. 110 : 1842-1857.

Pfurtscheller G, Neuper C, Pichler-Zalaudek K, Edlinger G, Lopes da Silva FH (2000). Do brain oscillations of different frequencies indicate interaction between cortical areas. Neurosci. Letters 286 : 66-68. 